\title{
LOGICAL RELATIVISM THROUGH LOGICAL CONTEXTS
}

\author{
Jonas R. Becker Arenhart ${ }^{1,2}$ \\ ${ }^{1}$ Federal University of Santa Catarina \\ ${ }^{2}$ Federal University of Maranhão
}

Original scientific article - Received: 06/07/2021 Accepted: 12/10/2021

\begin{abstract}
We advance an approach to logical contexts that grounds the claim that logic is a local matter: distinct contexts require distinct logics. The approach results from a concern about context individuation, and holds that a logic may be constitutive of a context or domain of application. We add a naturalistic component: distinct domains are more than mere technical curiosities; as intuitionistic mathematics testifies, some of the distinct forms of inference in different domains are actively pursued as legitimate fields of research in current mathematics, so, unless one is willing to revise the current scientific practice, generalism must go. The approach is advanced by discussing some tenets of a similar argument advanced by Shapiro, in the context of logic as models approach. In order to make our view more appealing, we reformulate a version of logic as models approach following naturalistic lines, and bring logic closer to the use of models in science.
\end{abstract}

Keywords: Classical logic; intuitionistic logic; relativism; logic as models; context constitution 


\section{Introduction}

Logical generalism, as the name suggests, is the thesis that logic is general. This is ambiguous in the same measure as the term 'logic' is: on the one hand, it may denote 'logic' as a discipline, on the other, it may denote 'logic' as a specific system of logic. As Shapiro notes,

Moreover, logic is ubiquitous. [...] there is a longstanding view, with a stellar pedigree, that logical consequence is topic neutral; it applies everywhere. Even if that is challenged, [...] it remains that every coherent perspective - every language, every form of life, every context - has a logic. (Shapiro 2014, 165)

Here, we shall not discuss whether logic, taken as a science, is general, or universal, or ubiquitous. Rather, we shall focus on the claim that logic is general when one considers distinct systems of logic attempting to capture the validity of inferences in natural language (the so-called 'canonical application'; see Priest 2006, 196-197). Our claim, again, is that distinct systems are required and legitimate for distinct contexts, even when the field of application concerns inferences in natural language.

One could complain about that way of framing the problem, which emphasizes the role of distinct systems of logic. It could be said that validity in distinct systems should not be confused with validity per se or validity tout court; certainly, the claim could go, distinct systems characterize distinct notions of the consequence relation, but that is not what is at stake in philosophical debates. Rather, what is being disputed is whether distinct notions of validity are legitimate, or correct. That is, the question is whether there are, out in the wild, different notions of validity that require distinct systems to be characterized, and of which these systems are said to give a correct/incorrect description (depending on the case).

Now, although one could advance such an objection, our discussion will not presuppose that there is such a thing as validity per se, or validity tout court. However, as we shall see, there is a sense to be made of claims of distinct systems being correct for distinct contexts. Basically, the notion of correctness, as our proposal will characterize it, does not require correspondence of a theoretically described notion of validity with an independently existing notion of validity, out in the wild (this will be discussed latter). Furthermore, given that we can only characterize the distinct notions of validity that are in dispute in terms of some logical theory, using the logical apparatuses furnished by distinct systems, we 
shall keep with our talk of distinct systems, and not talk in terms of validity per se. Certainly, much more could be said about the idea that logical correctness is related to the correspondence of a system of logic with an intuitive or pre-theoretical notion of validity, but this is enough as a warning to begin with. We shall avoid this notion in our discussion, given that a proper treatment of this problem would require a different route.

With those points out of the way, let us proceed. Our next task is to attribute a more precise meaning to the idea that logical theories, or logical systems, can be claimed to be general or local. There are some claims advanced to that purpose, although none of them provides a precise characterization that could be adopted as an official definition. As Routley $(1980,83)$ has advanced the claim of generalism, approvingly, what lies behind the generalist thesis is a worry about the scope of logic, the fact that "[1]ogic is not merely a local matter, and should, insofar as it is correct, apply universally." Notice that this connects correction and generality. The opposite of generalism, a form of localism, may be understood then as the claim that logic is a local matter; a logic may be correct only locally.

Some opponents of logical generalism go in the same direction when it comes to characterizing the core of the generalist thesis. Wyatt and Payette (2021, 4813), for example, characterize generalism as the claim according to which "logical systems and logical laws must have universal application". Dicher (Forthcoming, 2), also not a defender of generalism, characterizes generalism as consisting of the view that, on what concerns logic, "there are no exceptions to its laws, which apply across every domain of inquiry, irrespective of the particular features of that domain". Again, the most important feature of generalism concerns the claim that logic meets no borders; a system must have its inferences and validities applying in every context. Logic would be local, then, if its laws would have local applicability or validity, if distinct systems were required to account for distinct domains. Hjortland $(2013,356)$ frames the localist claim in terms of the existence of at least two domains of discourse for which correct deductive reasoning requires distinct logics.

What these characterizations have in common, together with the discussion on generalism is, the idea of a context, or a domain, along with the claim that logic must be correct, or applied properly, irrespective of the context, or domain. Generalism involves the claim that a logical theory applies in every context or domain, it is insensitive to the demands of each particular domain it may meet. The individuation of a domain, then, is granted independently of the underlying logic; that is, according to generalism, in specifying a domain or context, the underlying logic is taken for granted (given that it is universal, context-independent), and each domain builds 
over it with its specific features (the context-depend ones). According to generalism, what is common to all domains or contexts is the underlying logic.

Notice that one may regard that only one system of logic fits the bill of being general enough, of accounting for every domain, resulting in a monist position about logic, or else one may hold that distinct systems of logic are all equally successful in being general as required, resulting in pluralism about logic. The distinction general/local does not collapse into the distinction monism/pluralism, although it is much more common to find monists among generalists than pluralists. For localists, those that hold that distinct logics may be correct or appropriate for distinct contexts, the same distinction applies. Given a context, one may believe that only one system of logic is correct for that context (local monism, a position defended by da Costa 1997), or that a whole family of distinct systems may be equally correct for that context (local pluralism, a position defended by Bueno 2002, for instance).

The terminology thus introduced requires that we distinguish between the pair local/general on the one hand, and the pair one/many on the other, when it comes to logic. ${ }^{1}$ Their combinations give rise to the current spectrum of traditional positions: logical monism and logical pluralism, as traditionally understood, are generalist theses, holding that there is one and that there are many correct logics, respectively. Relativism or localism is the thesis that logic is local, and the question remains open as to whether there are many distinct logics for one context, or only one for each context.

In this paper, we shall focus on the general/local divide, leaving the issue of one/many for another occasion. Our plan is to elaborate over already existing proposals for logical relativism, and we do so by putting logic in a naturalistic setting in two related senses. First of all, naturalism is understood as the methodological claim that there is no first philosophy to judge science, with logic and mathematics understood as part of science. Second, the approach advanced here is naturalist also in the way that the 'logic as models' approach is framed, requiring that models be understood in closer connection to the workings of models in science; more specifically, we shall suggest that the understanding of models in science according to the view called 'models as epistemic tools', as developed by Knuuttila and Boon (2011), can be fruitfully adapted to the case of logic. This will provide us the appropriate understanding of 'context' required to motivate localism in logic. As we shall see, logical generalism is not

\footnotetext{
${ }^{1}$ This clearly complements the distinction advanced by Haack $(1978,223)$ and, following Haack, by Hjortland (2013, 356-357).
} 
motivated, if an account of logic that is more naturalistic is adopted, and when the notion of a context is properly understood in connection to scientific modeling. In order to motivate our proposal, we shall briefly discuss a related argument advanced by Shapiro (2014), who also defends the logic as models approach. We shall use what appear to be some tensions in Shapiro's approach to suggest an alternative account that not only overcomes the difficulties, but also presents some virtues that recommend it as a better option for the friends of the logic as models approach. As a kind of bonus, we hope, the resulting combination of naturalism and 'logic as models', as developed here, can be used to articulate a version of logical anti-exceptionalism; according to the latter, logic is continuous with empirical science in many respects (see Hjortland 2017). Perhaps, the view defended here does contribute to substantiate this claim, although we shall not develop it here.

Perhaps one more word on the pluralism/monism divide is in order. Typically, this is directly connected with the question of whether one or many logics are correct, and the problem of the correction of a logic is a substantial one, concerning connection of the formal systems with extrasystematic considerations about validity (see Haack 1978, chapter 2). As we shall propose in the paper, due to the kind of approach to logical contexts we advance, the 'correct' logic for a context becomes somehow an a priori issue, not open for substantial dispute (of course, the topic is developed in the paper). The locus of dispute, due to the naturalistic approach to the epistemology of logic and theory choice shifts, then, to the dispute on whether it is one or many logics that are currently required by the scientific community in its investigative practice. In this sense, the debate resembles the 'monism versus pluralism' debate, but the locus of importance is shifted, given that the issue of correction of a logic is mostly deflated. Developing this difference in depth would require a different paper, so that we just leave this as reminder for the reader. For those willing to keep the terminology, the view defended here would be classified as a form of local pluralism, although, again, the 'pluralism versus monism' debate is typically framed in terms that are considerably different from the one presented in the current paper.

The rest of the paper is structured as follows. In the next section, we advance Shapiro's argument against generalism, in the context of his approach to logic as models. In section 3, we present what may look like some difficulties for the strategy employed by Shapiro, and in particular, his understanding of the role of logic when it is considered under the logic as models approach. Our own suggestion arises as a solution to overcome the mentioned difficulties, and comes from a twist to Shapiro's perspectives. We argue that it combines perfectly with a more science- 
friendly approach to models in science in general, known in the philosophical literature as the 'logic as epistemic tools' approach. We conclude in section 5 .

\section{Shapiro's approach}

We start by presenting Shapiro's approach against logical generalism. As we understand it, it requires Shapiro's account of logic as per the logic as a model approach as a starting point. Basically, Shapiro holds that systems of logic are to be understood as models of inferences in natural language, in what is regarded as the same sense that 'model' is understood and employed in the sciences. This holds explicitly for formal languages:

I propose that a formal language is a mathematical model of a natural language in roughly the same sense as, say, a collection of point masses is a model of a system of physical objects, and a Turing machine is a mathematical model of a person following an algorithm, or perhaps a computing device. In other words, a formal language displays certain features of natural languages, while ignoring, simplifying, or idealizing other features [...]. (Shapiro 2014, 46)

Besides language, the modeling account also deals with the notion of logical consequence. The similarities of use of mathematical models in logic with the understanding of how models are used in other areas of investigation results in the question of the correctness of systems of logic being largely relative to our specific purposes, and to the accompanying claim that their success should be evaluated accordingly. As Shapiro claims:

With mathematical models, which features one focuses on, which are idealized, and which are ignored, depends on the purposes at hand, on why one is developing a model in the first place. Here, of course, our goal is to shed light on the relation (or relations) of logical consequence, and perhaps the norms for deductive reasoning and regulating beliefs to maintain consistency. So, presumably, in developing a logic-model, we should focus on and idealize those features of natural language that bear on deductive reasoning, or on regulating our beliefs for consistency, whatever those features may be. (Shapiro 2014, 47) 
This opens the door for arguing that distinct logics may be appropriate for distinct fields or contexts, given that we may have different purposes in different contexts. Indeed, this is the crucial ingredient for the kind of argument that Shapiro will advance for the relative character of logic. In order to do so, Shapiro couples this view on logic with a form of Hilbertianism on the philosophy of mathematics. Basically, this is an update on Hilbert's motto according to which, roughly speaking, consistency implies existence. That is, a mathematical structure that is consistent implicitly defines the entities it deals with, just as in Euclidean geometry the notion of point is defined implicitly by the geometrical axioms for 'point'. Given, however, that consistency is a matter of which logic one uses, and that distinct mathematical structures will end up being inconsistent when certain logics are adopted as their underlying logic, the result is that given our purpose of preserving consistency, perhaps distinct logics are required to account for the perceived consistency of distinct mathematical structures.

Notice how the dialectics to ensure relativism goes. First, it is assumed that a kind of mathematical pluralism holds. This means that distinct kinds of mathematical structures are legitimate due to their consistency and to their actual interest for mathematicians. As a second step, given this plurality, we may inquire over which logics are required to make such mathematical theories consistent, or, in other words, for which are the appropriate underlying logics of such theories. If it happens that distinct mathematical theories require distinct logics, then we are justified in adopting a form of localism about logic, that is, that distinct logics apply in distinct domains or contexts (Shapiro calls this relativism). Here, the logic as models approach is playing a major role: given the diversity of mathematical structures taken as legitimate as a kind of point of departure, or as a kind of 'neutral' data that appears to be independent of the issue of which logic or logics are appropriate, we idealize the inferential practices of existing distinct mathematical theories in order to comply with the demands of consistency in each case. The result, as claimed, is that distinct theories will end up requiring distinct logics if their internal consistency is to be preserved.

The case for the general argument is made with an illustration employing intuitionistic mathematics. As it is well-known, intuitionistic theories conflict with classical mathematics, and this conflict concerns the inferences available in each case (see Shapiro 2014, chapter 3, for specific examples concerning intuitionistic theories: Peano arithmetic using intuitionistic logic plus Church's thesis $(\mathrm{PA}+\mathrm{CT})$, the intuitionistic analysis, and smooth infinitesimal analysis (SIA)). Let's focus on the 
simple case of intuitionistic analysis. In classical analysis, developed using classical logic, it is possible to define real functions that are not continuous (this is widely known, of course). Intuitionistic analysis does not vindicate such a simple fact, and this surprises students of classical mathematics when they hear of it for the first time. How can that be? This is a direct result of the theory of real numbers adopted by intuitionists.

One of the sources for the difference is to be found in the very concept of real number in intuitionistic analysis. Indeed, the intuitionist may consider a real number as an equivalence class of Cauchy sequences of rational numbers, just as a classical mathematician does. However, a sequence, in this context, is a choice sequence, it is only potentially infinite, never complete (intuitionists do not accept complete infinities, remember). Recall that a sequence $s$ of rational numbers is Cauchy if for every rational number $\varepsilon>0$, there is a natural number $\mathrm{N}$, so that for every natural numbers $\mathrm{m}>\mathrm{N}$ and $\mathrm{n}>\mathrm{N},|\mathrm{s}(\mathrm{n})-\mathrm{s}(\mathrm{m})|<\varepsilon$ (intuitively, the terms of the sequence $\mathrm{s}$ may be seen as approaching each other, as the function picks as arguments numbers standing after a given $\mathrm{N}$ in the usual order). For an intuitionist, given any $\varepsilon$, if a sequence is Cauchy, one must be able to compute the $\mathrm{N}$ after which the members of the sequence are within the $\varepsilon$ given. Over such a view of real numbers, we have:

Brouwer's theorem: all real functions defined over a closed interval are uniformly continuous.

The details of the proof of the theorem need not concern us here. What is more relevant is that the result conflicts with classical analysis, and that Shapiro makes use of this fact to argue for the requirement of distinct logics for distinct mathematical structures. He argues that if we add the law of excluded middle to intuitionistic analysis, we are able to define functions that are not continuous. In this sense, then, Brouwer's theorem holds only in the presence of restrictions to classical logic; it requires intuitionistic logic. Classical logic is not consistent with it.

As a result, given that intuitionistic analysis is taken as a legitimate kind of mathematical structure (the initial data, recall) deserving to be developed and investigated, a kind of relativism about logic arises, due to the fact that distinct legitimate mathematical theories require distinct logics to be consistent (notice also the naturalistic bent, bringing mathematical practice to guide theory acceptability, rather than philosophical claims). The result is a restriction on the applicability of classical logic, as well as of intuitionistic logic. As Shapiro puts it: 
conceding that the law of excluded middle, and thus classical logic, is not universally valid. That is, classical logic is not correct in all discourses, about all subject matters, etc. The intuitionist is right about that much. (Shapiro 2014, 82)

That is, the conclusion is precisely a denial of generalism (given that Shapiro admits that excluded middle holds in classical analysis). Given that intuitionistic logic is required to account for part of that practice in intuitionistic analysis, it seems that intuitionistic logic is legitimate as the underlying logic of a domain of investigation. Of course, given that one is also assuming that classical structures require classical logic, then, distinct domains or contexts require distinct logics. This would justify rejection of the version of generalism we are concerned with.

\section{Some tensions for Shapiro's account}

Although, as it will become clear, we are in agreement with the main conclusion established by Shapiro, we still seem to find some sources of tension that must be acknowledged in Shapiro's path leading from Hilbertianism about mathematics to logical localism. In this section we shall bring some of them to the fore. Avoiding such tensions is the major goal of the approach we shall advance in the next section.

The first source of concern is related to the requirement of consistency preservation as a sign for the appropriateness of a logic for a given context. That is, to recognize that a logic is appropriate for a given context, one is required to check whether that logic preserves or grants the context's consistency (relative to that very same logic). Although it seems quite reasonable in the context of mathematical theories, the worry is that it may lead one to the wrong kind of account of the underlying logic in some quite interesting cases. There are historically well-known cases, such as Frege's Grundgesetze, where choice of the underlying logic is out of the question, but still, the system is not consistent. Still, despite its inconsistency and triviality, the system is not without logical interest. Also, for a more recent episode, da Costa's original formulation of his paraconsistent version of the set theory NF (New Foundations) was established as trivial, although there was no question of the choice of a logic (see da Costa 1986). Again, although the logic chosen by da Costa was not properly ensuring consistency (in this case, non-triviality), the system was clearly interesting, and had some important lessons to teach on the nature of paraconsistent set theory. 
As a result, the requirement of consistency does not seem to provide, in some cases, at least, the best help when it comes to connecting systems of logic with specific contexts. Some legitimate mathematical contexts can be said to have well determined logics that clearly violate the requirement. In other words, some contexts come with a logic, explicitly formulated, that violate the requirement of consistency. In these cases, it does not seem appropriate to hold that the logic leading to inconsistency/triviality did not contribute to the individuation of each context. They did, but it ended up being the case that the systems were inconsistent/trivial. The very idea that one can attempt to fix Frege's system, as neo-logicists do, or that da Costa could fix his system, only makes sense if we accept that the original system is and remain inconsistent/trivial. Accepting that the underlying logics help us characterize and individuate a context indicates that changing the underlying logic will result in a different theory (more on this soon).

Perhaps one could object to this point in the following way: the cases brought here do not cause a problem to Shapiro, given that Shapiro is only concerned with what are considered legitimate mathematical structures, that is, consistent contexts really investigated by the mathematical community. Being inconsistent, Frege's Grundgesteze is not legitimate; being trivial, da Costa's theory is not legitimate, and Shapiro would have nothing to do with them. However, it seems to us that this would limit the interest of Shapiro's approach, missing interesting facts about the comings and goings of mathematical structures. For example, consider Cantor's naive set theory. With the discovery of Russell's paradox, the theory was fixed in a plurality of alternative ways, and interest in it did not disappear due to inconsistency. So, in a sense, it may happen that some theories are individuated with logics that do not grant them consistency, or non triviality. However, that does not mean that such theories cannot be interesting from a mathematical point of view. Rather, people try to keep some of the results of the theory either by changing the logic, or by changing some of the axioms specific to the theory (in both cases, with new contexts arising). That is, some theories may be on the radar of mathematicians even if they are inconsistent, and the search for a consistent version may be even a part of the pursuit of such mathematicians.

The second perceived source of tension concerns the very idea of a context. Shapiro has offered the following characterization of a context:

I propose that each "context" includes a specific mathematical theory or structure. It would be the mathematical theory being advanced at any given time by a mathematician or a group of mathematicians. In line with the foregoing eclectic orientation, 
each such context has a specific logic: classical logic for the classical theories, intuitionistic logic for the intuitionistic ones, etc. Sometimes we will just think of a logic alone as a context, if the ambient mathematical theory is not in focus or does not matter. (Shapiro 2014, 89)

However, in inferring from mathematical pluralism to logical localism, one must acknowledge that the adoption of distinct logics is a result of distinct contexts being already considered as legitimate, which in this case are the distinct mathematical theories currently investigated by the mathematical community. But distinct mathematical theories (which are playing the role of the contexts, here) seem to be characterized as incorporating a logic beforehand. That is, a logic is part of what constitutes and individuates a context. Under these conditions, it seems implausible to think that we can have a context (when this is a mathematical theory) individuated independently of a logic, only afterwards extracting from it a logic. Alternatively, we could proceed as Shapiro implies, seeing logics as models, and attempting to model the inference patterns of the context in each case where the context is legitimate in the eyes of the mathematicians. That is, logics are there to begin with, characterizing the context, but also, must be extracted from the context. So, the dilemma may be put as follows: on the one hand, the logic as models approach requires that we somehow idealize from given practices of inferences, generating a set of inferential patterns considered appropriate for the goal of preserving consistency in that context. On the other hand, a context is specified with the help of a logic. But then, we seem to be in trouble: logic must be already there to define a context, and also, be extracted from a context by the modeling procedure. It seems that we cannot have it both ways.

Given that this issue is of central importance for our own argument against logical generalism, let us check what is going on in more detail. To motivate the failure of generalism, one must argue that distinct logics are required for distinct contexts. Shapiro attempts to grant that fact by starting with distinct mathematical theories that are playing the role of the contexts and provide a kind of neutral data on the issue of which logic is appropriate. Given these contexts, he proposes to somehow extract, by means of the modeling approach, the required logics that account for their consistency, by checking which system preserves the consistency of each mathematical theory. This would make a case for distinct logics in distinct contexts that is not question begging, and that confers credibility to the view, given that the distinct contexts one started with are scientifically respectable. However, when it comes to defining a context, systems of logic already play a role in their individuation. If this is really so, as it is suggested by the characterization of a context, then, one cannot really have a fully 
convincing argument for the failure of generalism, given that such distinct logics were admitted as legitimate right from the start, with the claim that distinct mathematical theories constitute distinct contexts, and that such theories come with a logic.

We can make the point quite forcefully considering Brouwer's theorem, mentioned earlier. The fact that it is proved in intuitionistically acceptable ways already points to the need of identifying some logical resources, and that the law of excluded middle is not one of them. This should give us pause to think that perhaps intuitionistic logic (or something quite similar) must be available in the background beforehand, otherwise the context would be developed very differently. At least when it comes to mathematical theories, it is quite difficult to think that one could have some inferential practices in developing the theory that latter, under closer analysis, turn out to be intuitionistic logic, without consciously applying them in order to develop the mathematical context to begin with. In this sense, logic is required to characterize the context. However, as we have seen, the move by Shapiro also seems to require that logic is established after the context is available, by some kind of modeling activity. There lies the tension.

One could hold that the tension is illusory. ${ }^{2}$ In fact, it can be argued that every context comes with an underlying logic L, but then, with the development of the theory, still inquire whether the theory is really consistent with L. It may turn out that it is, and that L is the best model for the kind of inference used in this context, or it may turn out that a distinct system of logic may be more adequate, resulting in the case that the logic discovered after the modeling process is applied, L', is different from L, but still, more appropriate than L. This, it could be claimed, could make the use of an underlying logic to individuate a context compatible with the use of the logic as models approach to obtain a logic from the context, dispelling the kind of trouble that we have attempted to point out. But notice that this objection cannot dispel the worries we have raised. Given that a logic $\mathrm{L}$ is presented as the underlying logic for a context, it would be odd, to say the least, to discover, afterwards, that we did not properly infer according to it, so that the modeling process of our inferences ended up delivering a different logic. Why start with $\mathrm{L}$, then, if we are not required to infer according to it? If it happens that the logic L leads us to triviality, such as in Frege's Grundgesetze case, then, of course, we can only discover that by really using the logic L. This allows us to fix the context, in case it is of mathematical interest, originating new contexts (as discussed earlier also in the case of Cantor's theory and paraconsistent set theory).

${ }^{2}$ Again, I owe the objection to an anonymous referee, to whom I would like to thank. 
As a result, we keep the claim that there is a tension in locating logic in the context to begin with, and also attempting to extract it from the context by use of the modeling approach. There are basically two views on the identity of context playing a major role here. On the one hand, a context is individuated by its underlying logic, so that the logic comes with the context. On the other hand, a context is given independently of a logic, so that the logic appropriate for the context is identified by a process of modeling of the inferential practices of users, restricted also to the demands of consistency. The argument from mathematical pluralism to logical localism depends on the latter view, it seems, because the plan is to infer the diversity of logics from the diversity of mathematics, by observing the demand of consistency.

One can avoid the difficulty either by attempting to define 'context' without the use of logic as a constitutive component, which seems difficult in this circumstance, or by providing for another account leading from distinct contexts to the acceptability or correctness of distinct logics in such contexts. Our proposal consists in following the second route, and we shall see logics as contributing to the individuation of contexts in a more thoroughly naturalistic approach.

\section{Inverting the perspective}

In order to avoid the tension mentioned in the previous section, due to the very nature of a context, we shall acknowledge right from the start that systems of logic do act as (at least partially) constituting contexts. That is, in our view, a logical theory contributes actively to the individuation of a context; contrarily to what the generalist suggests, that a fixed logic is taken for granted, and that the specific contents of a context are added on the top of it, we allow that even a system of logic may be used to legitimately individuate a context. As we shall argue from now on, this has at least two main advantages: it avoids the problem of an apparent kind of circularity in justifying the use of a logic in a given context, and also the problem of deciding issues of the right logic for a context (without requiring that there is a notion of validity per se, as mentioned at the beginning of the paper). These issues are solved by the more flexible notion of context that we advance.

It should be recognized that when it comes to mathematical theories, at least, logics are indeed part of the characterization of their respective contexts. To begin with the motivation for such a characterization of context, and the claim that it leads to localism quite directly, notice that 
this will already make a better sense of the currently developing literature of inconsistent mathematics. Even though inconsistent mathematics does not enjoy (at least for now) the same kind of wide acceptance of intuitionistic and classical mathematics, it is a field that has been growing in recent years. Consider the following definition of inconsistent mathematics:

Inconsistent mathematics is the study of the mathematical theories that result when classical mathematical axioms are asserted within the framework of a (non-classical) logic which can tolerate the presence of a contradiction without turning every sentence into a theorem. (Mortensen 2017)

In other words, inconsistent mathematics are the mathematical theories developed over paraconsistent logics (see also Priest 2006, chapter 10 and the definition of inconsistent arithmetic). This defines a family of contexts in which paraconsistent logics are the correct logics, by fiat, as it were. Clearly, distinct kinds of paraconsistent structures require distinct kinds of paraconsistent logics, and the logic must be clearly specified right from the start. Now, if other contexts may be defined in the same way, and are considered legitimate by anyone in the dispute, then, there is a good case against the generalist.

Before we proceed, notice how, in the case of inconsistent mathematics, such systems of logic are allowing us to individuate the contexts in question; inconsistent mathematics is defined as employing paraconsistent logics to begin with. In an important sense, there is no paraconsistent mathematics as a kind of activity first, and afterwards, we go on looking for the inferential patterns that enable such mathematics (that make it 'consistent', meaning 'non-trivial' here). The direction suggested by Shapiro, of going from the mathematics to the logic, would hardly work here. Rather, without such logics there to begin with, there would not be a case for the existence and complete understanding of the identity of such contexts. In the case of mathematical contexts, the logics are assumed by default, and they are the correct logics for the specific contexts they help individuate to begin with. In the same sense, we suggest, it would be odd to have intuitionistic mathematicians, and classical mathematicians too, proving theorems, each in his or her own domain, and only afterwards looking for their specific inferential patterns, in order to investigate which logic is more suitable. The patterns codifying valid inferences are not there somewhat hidden, awaiting to be found by a posteriori modeling activity. Rather, they are set at the beginning, to individuate the context. The logics act as enabling the development of the kind of mathematics of which they are the underlying logics. 
It is not the case, then, that distinct mathematical theories or structures make a case for the plausibility of the use of distinct logics; rather, distinct logics act to enable that distinct mathematics be developed. This solves the problem of determining the individuality of a context, avoiding what was perceived as a kind of circularity in the previous section. It puts the issue of the appropriate direction of the dependence of a context on logic on a clearer basis: the adoption of distinct logics is not a result of the acceptance of distinct mathematical structures as legitimate; rather, the distinct mathematical structures are a result of distinct approaches to logic, which act as a guide in the development of such mathematical structures. Although this may sound historically inaccurate in some cases, due to Brouwer's distrust of logic in general, there is a case to be made for it, even on what concerns intuitionism. Recall that although Brouwer did not develop a system of intuitionistic logic, his own approach to constructive mathematics originates in great part from his distrust of classical logic, and on restrictions to classical inference modes. In fact, in order to characterize constructive mathematics, in general, one needs to appeal to the kind of inferences, or logical behavior that is the basis of such contexts:

Constructive mathematics is distinguished from its traditional counterpart, classical mathematics, by the strict interpretation of the phrase "there exists" as "we can construct". In order to work constructively, we need to re-interpret not only the existential quantifier but all the logical connectives and quantifiers as instructions on how to construct a proof of the statement involving these logical expressions. (Bridges and Palmgreen 2018)

In this sense, just as inconsistent mathematics is mathematics developed over paraconsistent logics, constructive mathematics requires a constructive understanding of the logical apparatus to begin with; the logic contributes to the identity of the context. And we may go even further, and consider classical mathematics, which was here even before something like classical logic was available, right? How can it be that classical logic acts as enabling it? Well, notice that the epithet 'classical' was applied to classical mathematics only after classical logic consolidated. Classical logic is a recent invention, and a distinction between 'classical' mathematics and other types of mathematics is only available after the consolidation of classical logic. So, in this sense, the distinct logics and inference patterns required for distinct contexts, in the case of mathematical theories, are part of the very definition of a context, and are correct for those contexts due to this very fact. 
But now, given that the localist thesis is no longer inferred from a given neutral data, the plurality of mathematical theories, how do we grant that distinct logics are required for distinct contexts? Or, in other terms: how do we grant that distinct logics are required for distinct contexts, and that they are correct for them? We need to separate two distinct issues that are conflated in this kind of question. One way of looking at the question is concerned with the correctness of a logic for a specific context. This, at least in the case of some mathematical theories, is solved by the appropriate, and more refined, notion of a context that we advanced. A logic is already employed when it comes to characterizing a context, and is the appropriate logic for that context. After defining contexts like that, right from the start there is a logic that is doing the work of being the underlying logic. One could believe that this makes the issue of the correct logic rather uninteresting; in fact, this brings the disputes over the appropriate logic to a quick solution. ${ }^{3}$ However, although this may be seen as deflating some of the disputes over the correct logic, which may be seen as a virtue by some, it also shifts the locus of interest to another question: which such systems are interesting, or worthwhile pursuing?

This is in fact the second question that is conflated with the previous one. It concerns the respectability, from a scientific point of view, of each such context that may be advanced for the consideration of the scientific community. Classical mathematics clearly has an upper hand here, given its long intellectual tradition and successful application to empirical sciences. But intuitionistic mathematics is also an institutionally recognized scientific research program. Anyone ruling one of such contexts out would be adopting a revisionist program of the philosophy of mathematics that does not account for the practice of the discipline in our days, and as such would have the burden of proof.

This may be put in the context of the Carnapian principle of tolerance. The principle requires that we allow distinct systems to be investigated, and not to discard them based on philosophical prejudice. However, tolerance is still not enough to grant scientific respectability and ensure wide adoption of such systems in research programs. Tolerance concerns the fact that each one is free to advance a framework as something worth of pursuit; this, by itself, does not grant that the system will be pursued. Only science, as an institution, determines which systems (understood here as mathematical structures) are worth of investigation. ${ }^{4}$ Certainly, classical and intuitionistic mathematics pass this latter test. Given that each require

\footnotetext{
${ }^{3}$ Thanks to an anonymous referee for pointing that.

${ }^{4}$ Thus, logic and mathematics may also be seen as providing for research programs, in a Lakatosian sense, as suggested by Priest (1989).
} 
a distinct logic, a form of logical localism in current mathematical practice seems to be vindicated. In this sense, then, generalism fails, because it cannot account for the current state of mathematics. One can be generalist only at the price of rejecting mathematical practice, which is possible, but not totally recommendable from a naturalistic point of view.

The, in a sense, we suggest a division of labor between the question of correctness of a logic for a given context and the question of what makes a system an interesting object of research. In the picture suggested, although the question of correctness becomes deflated, there is still an issue of whether the diversity of systems available can become an integrating part of current scientific enterprise. From the relativist point of view advanced, a plurality of systems is justified in the measure that they are part of such an ongoing enterprise. As Caret proposed:

An honest naturalist simply takes mathematics as it stands and respects the autonomy of the discipline, rather than imposing outside ideas about how it 'should' be practiced. Who are we to police the bounds of mathematics because of some hangup about bivalence or truth-tables? (Caret 2021, 4964)

Such a practice recommends that some non-classical structures are currently part of the mathematical practice and this legitimates them. Notice that the issue of whether intuitionistic logic is correct for such practice is a prior issue (and here we differ from Caret); the point of relevance is accepting intuitionistic mathematics as part of the scientific enterprise. Again, this makes relativism interesting, the fact that it is anchored in the practice of science.

Let us contrast this approach again with Shapiro's strategy. While Shapiro uses the fact that our scientific community recognizes diverse mathematical structures as worthy of study and engagement as a starting point, which then leads to contexts and, from them, to distinct logics, we use logic as enabling the development of distinct mathematical theories, which, then, are acknowledged (or not) by our community as worthy of development (as fruitful research programs). That is, both approaches will have to appeal to the verdict of the scientific community on what concerns distinct mathematical structures and their scientific respectability as fruitful mathematical programs of research. However, while Shapiro uses this fact as a springboard to logical localism, attempting to ground the need for distinct logics in this fact, we use distinct logics to provide the very source of such distinct contexts. Scientific respectability comes after that, if it ever comes for some of the mathematical theories that are proposed. This describes perfectly well the situation of the inconsistent mathematics 
program: this is clearly a program where it is known, beforehand, which logic is the underlying logic of the enterprise. What friends of paraconsistent mathematics claim is that such structures are also worth of investigation, that the mathematical community should also join the efforts of developing such structures, due to theoretical rewards to be expected. Whether the mathematical community will listen to the call, time will tell, but it is largely an issue concerning the practice of mathematics, not of choice of the appropriate logic.

There are many advantages in reversing the approach to contexts as we have done. First, we have a clearer identity condition for contexts; mathematical theories are not entities awaiting for a logic to be attributed to them; rather, they are endowed from the start with prescriptions for the correct inferences. Second, this solves by default the issue that distinct logics are required for distinct contexts, basically, because the logic is already an ingredient of the context. Third, it is compatible with a version of the tolerance principle in which distinct logics may be used (as they indeed are, as the case of paraconsistent mathematics attests) to advance different contexts, which are then developed in the hope that the community may somehow recognize their importance.

The approach is very logic-oriented; it makes use of the fact that the very notion of 'domain' gets broadened with the rise of non-classical logics, and with the recognition that logics themselves may be used or required to characterize contexts. This allows for distinct logics being used in distinct contexts by fiat, something that could not be imagined when such distinct logics were not available. So, the anti-generalist has a somehow direct case once distinct logics are present to constitute distinct domains. The point is that the easy case can become also epistemically respectable when such contexts are also scientifically relevant, and this is what happens with the intuitionistic mathematics, for instance. This is as far as a naturalist would demand of justification for the distinct logics, that they be really part of current science, and is compatible with tolerance with the development of alternative approaches, which then will look for their place in the scientific enterprise.

We can finish now with a short discussion about how the logic as models approach suits in the picture, once this new understanding of context is adopted. Recall that we have suggested that logics help individuate a domain, instead of first having a domain or context, and then looking for the logic. In this sense, recall, the proposal is quite logic-centered, in the sense that it allows that logics may contribute to inform, in a sense, the nature of a domain or context to which they are applied. This also means that a logic and a context are not independent entities, awaiting to be 
matched. Rather, the logic somehow contributes to give a more specific shape and identity to the field of its own application. In more general terms, then, in the picture being proposed here, one needs an account of models that sees models as contributing actively to the character of the target they are intended to apply to. Typical accounts of models do not see models as having so much to offer on the way to individuate their targets or contexts of applications; rather, the typical accounts focus on the relation of models and targets, as two independent entities.

This situation reflects itself in the fact that most accounts of the role of models are still very much focused on the representation relation. The plan is that there are models on the one side, and targets on the other, and that knowledge about the target is obtained when the models are properly related to their targets. These accounts all recognize the role of abstraction, idealization and simplifications, but still, this is not enough to precisely account for the epistemic role that models play in our scientific activities:

Apart from simplifications, approximations and idealizations, scientific modelling involves significant conceptual work, which covers such epistemic activities as discerning specific types of phenomena, conceptualizing 'non-directly observable' objects, properties, or processes, and bringing phenomena under specific types of 'non-empirical' theoretical principles or concepts. It is difficult to see how these conceptual activities would fit into the traditional representational picture. (Knuuttila and Boon 2011,313)

That is, the traditional accounts (the 'representational picture' mentioned by Knuuttila and Boon) fall short of providing for a detailed enough picture of modeling. In particular, they fail to acknowledge the role of models in enabling the investigation of the target.

Luckily, there are proposals in the literature on the use of models in science that bring the required constitutive-enabling relation of the models to their targets to the center of the stage. Here, we shall propose that one may adapt the 'models as epistemic tools', advanced by Knuuttila and Boon (2011) to the case of logic, and get a result that is quite connected with the proposal we have been describing for the localist picture in logic. This account of models allows that a model play an active role in individuating the context in which they apply to; modeling involves more than just matching a model and a preexisting target. Rather, the modeling activity has a creative part in enabling that one investigates the target, because the target only gets available in precise terms through the applications of the conceptual machinery provided by the model; models and their targets are, in a sense, 
co-created. We see the target through the lenses of the model, as it were, and the justification of the model is partly built-in the model, given that the target is framed in theoretical language of the model too: ${ }^{5}$

in this activity of modelling, the construction of models is intertwined with the construction of new phenomena, theoretical principles and scientific concepts. As a consequence, the justification of a model is partly built into it in the process of modelling, implying that the representational approach, despite its focus on justification, fails to pay enough attention on how models are justified in scientific practice. (Knuuttila and Boon 2011, 311)

In the case of logics, as we have argued, use of a specific modeling of the inferences allowed enables the development of intuitionistic structures (and something similar may be said of classical mathematics, and inconsistent mathematics, with their respective logics). The justification for the use of a given logic is the fact that it is there to begin with, helping us to construct part of the phenomena to be accounted for; the models "both motivate and enable" the construction of the phenomena (Knuutila and Boon 2011, 317). The logics, understood as modeling kinds of inferences, motivate and enable the development of the mathematics associated with them. The same could also be said of classical mathematics, which, in the foundations period, needed to be put in firm basis, by following the standards of the newly developed classical logics. The individuation of the target depends in large measure of the logic used to model the inferences one is interested in. As it happens in science,

modelling typically involves a theoretical (re)description of the target phenomenon as well as the development of theoretical principles and scientific concepts. The model in the process of its construction functions as an integrating tool as well as a scaffold for further scientific reasoning. In this way the model serves also as a tool of its own development. (Knuuttila and Boon 2011, 316)

In this sense, the development of classical and intuitionistic logics explored the already available knowledge (the controversies on the validity of determined inferences, and the consequences of using only constructive inferences in proofs, for instance), to both be constructed and shape the field being modeled. That is, the model is not only a result of the data we

\footnotetext{
${ }^{5}$ The idea that models do incorporate 'built-in' justification for suiting their targets comes from Boumans (1999).
} 
put in it, but also it helps us in interpreting and somehow shaping the data, enabling further investigation in terms of the model. This is what happens in classical and intuitionistic mathematics. This is what happens in classical and intuitionistic mathematics. Some of the inferences used in the mathematical practices of the end of the nineteenth century have led to constructions of distinct approaches to the legitimate reasoning in mathematics and, as a result, these advances have enabled the development of distinct mathematical practices itself. The model of inferences is what ends up constraining the development of the field of investigation. The model acts so that it works to delimit the field of application, its phenomena.

Certainly, this only indicates in general lines how a 'logic as models' approach could go, but it does already give us a clear idea that the understanding of context we have suggested can be backed by an approach to models fine-tuned with the current understanding of models in science (being, thus, a naturalistic approach to the methodology of logic too). We suggest that a more pragmatic approach to models in science, which takes seriously the claim that the phenomena is theoretically laden, elaborated in theoretical terms furnished by the model, can have a lot of benefits for logic too. In particular, it can account for the fact that logics are used to generate a plurality of contexts, some of which may be of mathematical interest. Developing further the notion of logic as models in this specific approach is something we leave for some future work.

\section{Concluding remarks}

We have suggested that Shapiro's approach against a version of generalism in logic seems to face difficulties. We have identified that the major problem seems to be located in an ambiguity as to the role of logic in its relation to the domain or context where the logic is applied. Logic seems to be both used to characterize the context, and to be somehow extracted from the context. Our proposal to overcome the difficulty consists in locating logic right from the start as an ingredient constituting the domain or context. This makes full sense in the case of mathematical theories, at least as we now conceive of them (and we have discussed only the case of mathematical theories here). Not only does this dissolve the tensions in Shapiro's approach, but also makes room for a more naturalistic approach to the philosophy of logic.

As a by-product, we needed not to enter the discussion of how to grant that a given system is the correct choice for a given context, with disputes typically boiling down to issues of adequacy of systems of logic to the data. 
That is, we avoid the kind of discussion concerning whether a logic is right by relating a logic and a preexisting domain, both typically taken as being able to be characterized independently of each other. In our proposal, the correctness of a system is somehow built-in in the very context, and the idea that a context, which is gained so easily, deserves to be studied, depends on pragmatic factors; the decision on which systems are worth of study and development comes from science. Here, of course, Carnapian tolerance is playing a major role.

Furthermore, this approach is nicely suited to the view of logic as models, when 'models' are understood in more naturalistic terms. The view of logic as modeling inferences, and the inferences modeled as delimiting and individuating the field where they apply squares nicely with the localist picture we have advanced. In fact, it boosts the localist proposal advanced here. Advocating a generalist picture, according to this view, would require that one adopts a restrictive position on the domains allowed for an investigation, a restrictive view that is not easy to justify, and that is not justified in the current state of the art of the logic and mathematics as we find it. In this sense, the view advanced here not only helps us advance a more coherent form of localism, but also provides for a clear picture of how new domains come to be proposed, such as paraconsistent mathematics, as we have argued. Certainly, more would still be required to articulate the proposal in all its details, and one may still draw many more important lessons for the epistemology of logic from the use of models in more naturalistic ways, as suggested by the 'models as epistemic tools approach', when connected to the 'logic as models approach', but we leave this issue for another occasion.

\section{Acknowledgments}

The paper was prepared with the benefit of a Capes-Humboldt Experienced Researcher Fellowship, held at the Ruhr-Universität Bochum, Germany. The author is partially supported by CNPq (Brazilian National Research Council).

\section{REFERENCES}

Boumans, Marcel. 1999. 'Built-in Justification'. In Models as Mediators. Perspectives on Natural and Social Science, edited by M. S. Morgan and M. Morrison, 66-96. Cambridge: Cambridge University Press. https://doi.org/10.1017/cbo9780511660108.005 
Bridges, Douglas, and Erik Palmgren. 2018. Constructive Mathematics. The Stanford Encyclopedia of Philosophy, edited by Edward N. Zalta. Accessed November 1, 2020.

https://plato.stanford.edu/archives/sum2018/entries/ mathematics-constructive/.

Bueno, Otávio. 2002. 'Can a Paraconsistent Theorist be a Logical Monist?' In Paraconsistency: The Logical Way to the Inconsistent, edited by W. Carnielli, M. Coniglio and I. L. D'Ottaviano, 535-552. New York: Marcel Dekker. https://doi.org/10.1201/9780203910139-33

Caret, Colin R. 2021. 'Why Logical Pluralism?' Synthese 198: 4947-4968. https://doi.org/10.1007/s11229-019-02132-w.

da Costa, Newton C. A. 1986. 'On Paraconsistent Set Theory'. Logique et Analyse, 29 (115): 361-371.

da Costa, Newton. C. A. 1997. Logiques Classiques et Non Classiques. Essai sur les Fondements de la Logique. Paris: Masson.

Dicher, Bogdan. forthcoming. 'Requiem for Logical Nihilism; Or, Logical Nihilism Annihilated'. Synthese. https://doi.org/10.1007/s11229-019-02510-4.

Haack, Susan. 1978. Philosophy of Logics. Cambridge: Cambridge University Press.

https://doi.org/10.1017/CBO9780511812866

Hjortland, Ole Thomasson. 2013. 'Logical Pluralism, Meaning Variance, and Verbal Disputes'. Australasian Journal of Philosophy 91 (2): 355-373.

Hjortland, Ole Thomason. 2017. 'Anti-exceptionalism about Logic'. Philosophical studies 174, 631-658.

https://doi.org/10.1007/s11098-016-0701-8

Knuuttila, Tarja, and Mieke Boon. 2011. 'How do Models give us Knowledge? The Case of Carnot's Ideal Heat Engine'. European Journal for Philosophy of Science 1: 309-334. https://doi.org/10.1007/s13194-011-0029-3.

Mortensen, Chris. 2017. Inconsistent Mathematics. The Stanford Encyclopedia of Philosophy, edited by Edward N. Zalta. Accessed November 1, 2020. https://plato.stanford.edu/archives/fall2017/entries/mathematics -inconsistent/

Priest, Graham. 1989. 'Classical Logic Aufgehoben'. In Paraconsistent Logic. Essays on the Inconsistent, edited by G. Priest, R. Routley and J. Norman, chapter 4. Munich: Philosophia Verlag.

Priest, Graham. 2006. Doubt Truth to be a Liar. Oxford: Oxford University Press. DOI: 10.1093/0199263280.001.0001 
Routley, Richard. 1980. 'The Choice of Logical Foundations: Nonclassical Choices and the Ultralogical Choice'. Studia Logica 39 (1): 77-98. https://doi.org/10.1007/bf00373098

Shapiro, Stewart. 2014. Varieties of Logic. Oxford: Oxford University Press.

https://doi.org/10.1093/acprof:oso/9780199696529.001.0001

Wyatt, Nicolle; Payette, Gillman. 2021, 'Against Logical Generalism'. Synthese 198: 4813-4830.

https://doi.org/10.1007/s11229-018-02073-w 